\title{
Alterations of Thyroid Microbiota Across Different Thyroid Microhabitats in Patients With Thyroid Carcinoma
}

\section{Daofeng Dai}

First Affiliated Hospital of Nanchang University

\section{Yang Yan}

Zhejiang Provincial First Hospital: Zhejiang University School of Medicine First Affiliated Hospital

\section{Yong Yang}

Jiangxi Provincial People's Hospital affiliated to nanchang university

\section{Tianfeng Dang}

First Affiliated Hospital of Nanchang University

\section{Jiansheng Xiao}

First Affiliated Hospital of Nanchang University

\section{Weibin Wang}

First Hospital of Zhejiang Province: Zhejiang University School of Medicine First Affiliated Hospital

\section{Lisong Teng}

First Hospital of Zhejiang Province: Zhejiang University School of Medicine First Affiliated Hospital https://orcid.org/0000-0001-6470-9017

Jing Ye

First Affiliated Hospital of Nanchang University

Hongqun Jiang ( $\sim$ jianghq@ncu.edu.cn )

Affiliated Hospital of Nanchang University

\section{Research Article}

Keywords: Thyroid cancer, Microbiome, Lymph node metastasis, Biomarker, Sphingomonas

Posted Date: October 21st, 2021

DOI: https://doi.org/10.21203/rs.3.rs-967650/v1

License: (c) (1) This work is licensed under a Creative Commons Attribution 4.0 International License.

Read Full License 
Version of Record: A version of this preprint was published at Journal of Translational Medicine on November 30th, 2021. See the published version at https://doi.org/10.1186/s12967-021-03167-9. 


\section{9} 0 21

\section{Alterations of thyroid microbiota across different thyroid microhabitats in patients with thyroid carcinoma}

Daofeng Dai ${ }^{1+}$, Yan Yang ${ }^{2+}$, Yong Yang ${ }^{3+}$, Tianfeng Dang ${ }^{1}$, Jiansheng Xiao ${ }^{4}$, Weibin

Wang $^{2}$, Lisong Teng ${ }^{2 *}$, Jing $\mathrm{Ye}^{1^{* *}}$, Hongqun Jiang ${ }^{1 *}$

*Correspondence: 1steng@zju.edu.cn; yjholly@email.ncu.edu.cn;

jianghq@ncu.edu.cn.

${ }^{\dagger}$ Daofeng Dai, Yan Yang and Yong Yang contributed equally to this article.

${ }^{1}$ Jiangxi Otorhinolaryngology Head and Neck Surgery Institute, Department of

Otorhinolaryngology-Head and Neck Surgery, The First Affiliated Hospital of

Nanchang University, Nanchang, China

Full list of author information is available at the end of the article

(1)

\section{(1)}

.




\section{Abstract}

Background: In recent years, the incidence rate of Thyroid carcinoma(TC) has been increasing worldwide. Thus, research on factors of TC carcinogenesis may promote TC prevention and decrease the incidence rate. There are several studies targeting the correlation between gut microbiota and thyroid disease. Carcinogenesis of several malignancies is influenced by microbiota. However, thyroid microbiome of TC has not been revealed. This study investigated thyroid microbiota in different TC microhabitats.

Methods: We performed 16s rRNA gene sequencing using tumor tissues and matched peritumor tissues from 30 patients with TC to characterize thyroid microbiota.

Results: The richness and diversity of thyroid microbiota were lower in TC tumor samples than in matched peritumor tissues. At the genus level, the core microbiota of thyroid included Sphingomonas, Comamonas, Acinetobacter, Pseudomonas, Microvirgula, and Soonwooa. The abundance of Sphingomonas and Aeromonas was significantly increased in tumor tissues, while the abundance of Comamonas, Acinetobacter, and Peptostreptococcus was significantly enhanced in peritumor tissues. The combination of Comamonas and Sphingomonas could discriminate tumor samples from peritumor samples with an area under the curve (AUC) of 0.981 (95\% confidence interval [CI]: 0.949-1.000). The abundance of Sphingomonas was significantly higher in N1 stage than in N0 stage. Sphingomonas could distinguish between N0 and N1 stage with an AUC of 0.964 (95\% CI: 0.907-1.000).

Conclusions: The microbial diversity and composition were significantly different between peritumor and tumor microhabitats from patients with TC, which may eventually affect TC carcinogenesis and progression. The combination of Comamonas and Sphingomonas could serve as a powerful biomarker for discrimination between tumor and peritumor tissues. Furthermore, the higher abundance of Sphingomonas was correlated with lymph node metastasis, indicating that it may play a role in promoting TC progression. 


\section{Introduction}

Thyroid carcinoma (TC) is a common endocrine malignancy, with an estimated 567,000 new cancer cases and 41,000 deaths worldwide in 2018 [1]. The main histological types include papillary thyroid carcinoma (PTC), follicular thyroid carcinoma (FTC), Hurthle cell thyroid carcinoma (HCTC), medullary thyroid carcinoma (MTC), and anaplastic thyroid carcinoma (ATC), which account for $80.2 \%$, $11.4 \%, 3.1 \%, 3.5 \%$, and $1.7 \%$ of thyroid cancer, respectively [2]. In recent years, the incidence rate of TC has been increasing worldwide. Thus, research on factors of TC carcinogenesis may promote TC prevention and decrease the incidence rate.

Several organs, such as lungs, bladder, and urethra, have long been considered sterile. The advent of next-generation sequencing reveals that these organs are inhabited by a robust microbiota $[3,4]$. Due to the acidic environment of the human stomach, researchers previously believed that the stomach was not suitable for the growth of other microorganisms and was inhabited exclusively by Helicobacter pylori. However, recent advances in sequencing technology make it clear that the stomach is colonized by a huge number of microorganisms [5]. Dysbiosis of gastric microbiota can affect metabolism, inflammation, immunity [6,7], and eventually result in gastric cancer [8]. The imbalance between the types of microorganisms within the lung can cause lung diseases, such as cystic fibrosis [9], asthma [10], chronic obstructive pulmonary diseases [11], or even lung cancer [12]. However, the profile and functional role of thyroid microbiome in patients with TC has not been revealed.

There are several studies targeting the correlation between gut microbiota and thyroid disease. A study reported that the proportions of Pasteurellaceae and Prevotellaceae were higher, but the proportions of Veillonellaceae, Enterobacteriaceae, and Rikenellaceae were significantly lower in patients with Graves' disease compared to controls [13]. The abundance of Lactobacillaceae and Bifidobacteria was reduced, but the abundance of Enterococcus spp. was increased in hyperthyroid patients compared to healthy controls [14]. Su et al. reported that they 
observed significant differences in alpha and beta diversities of gut microbiota between patients with primary hypothyroidism and healthy individuals [15]. The fecal microbiota transplantation showed that total thyroxine levels were decreased in mice receiving the transplant from patients with primary hypothyroidism. Gut microbiota diversity and composition were significantly different between patients with TC and healthy controls [16].

The term 'thyrogastric syndrome' referring to the link between the gastrointestinal tract and the thyroid has been postulated in 1950s [17]. Gastric mucosal cells and thyroid follicular cells have the same embryonic origin because the thyroid gland develops from primitive gut cells [18]. Thus, we hypothesize that thyroid gland may also be colonized by microorganisms. For the first time, we performed 16s rRNA gene sequencing using tumor tissues and matched peritumor tissues from 30 patients with TC to characterize the core microbiota of thyroid, compare microbial diversity and composition of tumor tissues and matched peritumor tissues, identify differential taxa between tumor tissues and matched peritumor tissues, and characterize the microbial biomarkers for discrimination between tumor and peritumor tissues. The correlation between microbiota of thyroid carcinoma and clinicopathological factors was analyzed.

\section{Methods}

This retrospective study included 55 TC patients who underwent total thyroidectomy between March 2018 and December 2018 at the First Affiliated Hospital, School of Medicine, Zhejiang University. Two patients with body mass index $(\mathrm{BMI})>30$, two patients with history of malignancy or receiving radiotherapy/chemotherapy before operation, ten patients with recent usage of antibiotics, probiotics, prebiotics, symbiotics, and eleven patients with no paired tissues were excluded (Figure 1). Finally, 30 subjects were obtained for analysis of thyroid microbiota. The tumor and peritumor (about $3 \mathrm{~cm}$ adjacent to the cancer tissue) tissues were collected, which were confirmed by pathological diagnosis. Archival slides of patients were evaluated by two pathologists. We obtained the following clinicopathological information: 
gender, age upon diagnosis, tumor size, extrathyroidal extension, recurrence risk stratification, and clinical stage. TNM staging was determined based on the 8th edition of the American Joint Committee on Cancer staging system. The clinicopathological information was supplied in Table 1. Approval for this study was obtained from Medical Ethics Committee of the First Affiliated Hospital, School of Medicine, Zhejiang University. Patients signed an informed consent.

\section{DNA Extraction, Amplicon Library Construction, and Sequencing}

The genomic DNA of thyroid tissues (about $100 \mathrm{mg}$ ) was extracted using cetyltrimethylammonium bromide/sodium dodecyl sulfate method. To evaluate environmental contamination, six sterile Petri dishes with sterile water and sterile filter paper were placed in different corners of the operating room for 24 hours. The filter paper was transferred to sterile tubes for DNA extraction and subsequent PCR. The extracted DNA from the filter paper was used as quality control (QC). DNA integrity was analyzed by $1 \%$ agarose gel electrophoresis. DNA concentration and purity were verified using Nanodrop 2000 (Thermo). We used nested PCR to amplify the V3-V4 region of bacterial 16s rRNA gene. During the first round, the 16s rRNA gene was amplified using the 27F (5'-AGAGTTTGATCCTGGCTCAG-3') and 1492R (5'-GGTTACCTTGTTACGACTT-3') primers. In the second round, V3-V4 region of 16S rRNA gene was amplified using the 341F (5'-TCGTCGGCAGCGT CAGATGTGTATAAGAGACAGCCTACGGGNGGCWGCAG-3') and 806R (5'-G TCTCGTGGGCTCGGAGATGTGTATAAGAGACAGGACTACHVGGGTATCTA ATCC-3') primers. All PCR reactions were carried out in $25 \mu \mathrm{L}$ reaction mixture containing $10 \mathrm{ng}$ of template DNA. Barcode was added using index PCR (Nextera XT Index Kit v2, illumina). The PCR condition of DNA from filter paper was the same as that of DNA from tissues. Index PCR products were sequenced with the Miseq platform.

\section{Sequencing Data Analysis}

The raw reads were filtered to obtain the high-quality clean reads using USEARCH 
8.0. Chimera sequences were detected and removed using the UCHIME algorithm software [19]. Sequences with more than 97\% similarity were allocated to one operational taxonomic unit (OTU) using UPARSE software [20]. The phylogenetic affiliation of each 16S rRNA gene sequence was analyzed by RDP Classifier against the Silva 16S rRNA database using confidence threshold of $70 \%$. Subsequent analyses, including alpha diversity analysis, beta diversity analysis on Bray-Curtis distance, the linear discriminant analysis (LDA) effect size (LEfSe), and random forest analysis, were performed using MicrobiomeAnalyst [21].

\section{Statistical Analysis}

The statistical Analyses were performed using GraphPad Prism (Version 8.0; GraphPad Software) software. Statistical significance was defined as a two-sided $P$-value of $<0.05$. The Mann-Whitney $U$ test was used to calculate the difference in Chaol index, Shannon index, and the abundance of taxa between two groups.

\section{Results}

\section{Differences in Microbial Diversity and Composition Between TC Peritumor and} Tumor Tissues

As shown in Table S1, the 16s rRNA gene sequencing produced a median of 41778 reads for QC samples, 30 paired tumor and peritumor tissues. First, we analyzed alpha diversity to investigate microbial diversity between TC peritumor and tumor tissues. The Chaol index reflecting species richness was lower in tumor tissues than in peritumor tissues; however, the difference was not significant $(P=0.268$, Figure 2A). The Shannon index, which measures species richness and evenness, was significantly lower in tumor tissues in comparison to peritumor tissues $(P<0.001$, Figure 2B). To exclude the possibility of contamination from the environment, six QC samples were obtained from the operating room where the tissue samples were collected. The analysis of alpha diversity showed that the Chaol index and the Shannon index were both significantly lower in QC group compared with peritumor and tumor tissues $(P<$ 0.001, Figure 2A and B).

To compare the composition of the microbial community between peritumor and 
tumor tissues, beta diversity was analyzed using Bray-Curtis method, and Principal coordinate analysis (PCoA) was performed, which showed that significant clustering was detected between $\mathrm{QC}$ and tissues samples. The PCoA also indicated that peritumor and tumor tissues showed two distinct clusters (PERMANOVA, $R^{2}=0.330$, $P<0.001$, Figure 2C).

The taxonomic profiles of thyroid microbiota are shown in Figure 2D and E. We defined the core microbiota of thyroid tissues if it is observed in $80 \%$ of samples. At the phylum level, the core microbiota of thyroid was Proteobacteria, Bacteroidetes, Firmicutes (Figure 2D, Table S2 and S3). At the genus level, the core microbiota of thyroid included Comamonas, Acinetobacter, Chryseobacterium, Pseudomonas, Microvirgula, Soonwooa, Sphingomonas (Figure 2E, Table S4 and S5). The proportions of Comamonas, Acinetobacter, Microvirgula, and Soonwooa were lower in tumor tissues than in peritumor tissues (Figure 2E). The tumor tissues had higher abundance of Sphingomonas compared with peritumor tissues (Figure 2E). At the genus level, the core microbiota of QC samples included Enterobacter, Citrobacter, and Chryseobacterium, which were very different from those of thyroid (Figure 2E).

To exclude the effect of environment on thyroid microbiota, we eliminated OTUs annotated as Enterobacter, Citrobacter, and Chryseobacterium from TC peritumor and tumor tissues. The Chaol index was higher in tumor tissues than in peritumor tissues; however, the difference was not significant $(P=0.224$, Figure 3A). The Shannon index was significantly lower in tumor tissues than in peritumor tissues $(P=$ 0.022, Figure 3B). PCoA showed that significant clustering was detected between tumor and peritumor tissues (PERMANOVA, $R^{2}=0.162, P<0.001$, Figure 3C). At the phylum level, the core microbiota of thyroid was Proteobacteria, Bacteroidetes, Firmicutes (Figure 3D). At the genus level, the core microbiota of thyroid included Sphingomonas, Comamonas, Acinetobacter, Pseudomonas, Microvirgula, and Soonwooa (Figure 3E).

\section{Determination of Differential Taxa Between Different Thyroid Microhabitats}

To identify discriminative taxa between TC peritumor and tumor tissues, we analyzed 
198 the compositions of thyroid microbiota in peritumor and tumor tissues using linear discriminant analysis (LDA) effect size (LEfSe) method (LDA > 3.0, corrected $P$ value $<0.05)$. At the phylum level, we did not identify any differential taxa. At the genus level, the abundance of Sphingomonas and Aeromonas was significantly increased in peritumor tissues, whereas the abundance of Comamonas, Acinetobacter, Peptostreptococcus, and Proteus was significantly increased in tumor tissues (Figure 4A). The 5 differential taxa including Sphingomonas, Aeromonas Comamonas, Acinetobacter and Peptostreptococcus were also confirmed by the random forest analysis which revealed 10 differential taxa between peritumor and tumor tissues (Figure 4B). We further analyzed the differential abundance of the 5 discriminative features at the genus level between the two groups using the Mann-Whitney U test, and demonstrated that the abundance of these taxa was significantly different between the two groups $(P<0.05$, Figure $4 \mathbf{C}-\mathbf{G})$.

\section{Identification of Different Thyroid Microhabitats Based on Thyroid Microbiota}

Receiver operating characteristic (ROC) curve analysis was performed to evaluate the diagnostic value of the 5 differential taxa in distinguishing tumor samples from peritumor samples. The areas under the curve (AUCs) of the 5 taxa ranged from 0.746 to 0.884 (Figure 5). Comamonas and Sphingomonas had an AUC value $>0.800$ (Figure 5C and 5E), and were further selected as the potential biomarkers. The combination of Comamonas and Sphingomonas could rapidly increase the diagnostic accuracy in discriminating tumor samples from peritumor samples with an AUC value of 0.981 (95\% confidence interval [CI]: 0.949-1.000, Figure 5F).

To reveal the association between lymph node metastasis and thyroid microbiome, we analyzed microbial differences between tumor tissues from patients at N0 and N1 stage. The Chao1 index was significantly higher in N1 stage than in N0 stage $(P=$ 0.049, Figure 6A), while the Shannon index was significantly lower in N1 stage in comparison to N0 stage $(P=0.020$, Figure 6B). PCoA analysis based on the 
Bray-Curtis method showed that significant clustering was detected between patients at N0 and N1 stage (PERMANOVA, $\mathrm{R}^{2}=0.164, P<0.001$, Figure 6C). The LEfSe analysis showed that the abundance of Sphingomonas was significantly increased in N1 stage compared to N0 stage (Figure 6D), which was also confirmed by the Mann-Whitney $U$ test $(P<0.001$, Figure 6E). ROC curve analysis showed that Sphingomonas could distinguish between patients at N0 and N1 stage with an AUC of 0.964 (95\% CI: 0.907-1.000, Figure 6F). However, we observed no significant differences in Chaol and Shannon indices between male and female patients $(P=$ 0.349, Figure 6G; $P=0.657$, Figure 6H). PCoA analysis suggested that there was no significant difference in composition of thyroid microbiota between male and female patients (PERMANOVA, $\mathrm{R}^{2}=0.033, P=0.473$, Figure 6I). LEfSe analysis revealed no differential taxa between male and female patients. Figure 6J-L showed that there was no difference in the diversity and composition of thyroid microbiota between patients aged $\geq 55$ and $<55$.

\section{Discussion}

In this study, we used 16s rRNA gene sequencing to characterize thyroid microbiota in different thyroid microhabitats. The alpha diversity and beta diversity were both significantly different between QC samples and thyroid tissue samples, indicating that thyroid tissues were not contaminated by the surrounding environment. We found that TC tumor tissues had lower thyroid microbiota richness and diversity than matched peritumor tissues. A decrease in microbiota diversity in tumor tissues was also observed in patients with lung cancer and gastric cancer [22,23]. Nevertheless, an increase in gut microbiota diversity is observed in patients with TC, Hashimoto's thyroiditis, and hyperthyroidism [14][16][24] . At the genus level, the core microbiota of thyroid tissues included Comamonas, Acinetobacter, Pseudomonas, Microvirgula, Soonwooa, and Sphingomonas, while the core gut microbiota of TC encompassed Faecalibacterium, Bacteroides, Blautia, Rosebulia, Dialister, and Lachnoclostridium. These results showed that the composition of thyroid microbiota and gut microbiota of TC patients were different. 
We identified 5 differential taxa, including the genus Sphingomonas and Aeromonas enriched in peritumor tissues, and Comamonas, Acinetobacter and Peptostreptococcus enriched in tumor tissues. However, the comparison of the gut microbial compositions between TC and healthy subjects showed that 27 genera, including Bacteroides, Roseburia, Megamonas, Klebsiella, Blautia, etc., markedly differed between the two groups with significantly differential abundance. These results demonstrated that the differential taxa of thyroid microbiota and gut microbiota were quite different, indicating different roles of thyroid microbiota and gut microbiota in promoting TC development.

More importantly, the higher abundance of Sphingomonas was associated with lymph node metastasis, indicating its role in promoting TC development. An analysis of the global mucosa-associated microbiota revealed that the abundance of Sphingomonas was found to be increased in patients suffering colitis associated cancer compared with those with sporadic cancer [25]. Jeong et al. reported that the higher level of Comamonas in tumor tissues was associated with more metastasized lymph nodes in pancreatic cancer[26]. Comamonas is a cellulolytic microbe that could impact the host metabolism in cancer patients and play a role in inflammation [27]. Ling et al. reported that Comamonas was negatively associated with BDCA2+pDCs, indicating its correlation with antitumor immunity [28]. These studies showed that Comamonas and Sphingomonas may play important roles in tumor progression. Furthermore, we found that the combination of Comamonas and Sphingomonas could discriminate tumor samples from peritumor samples with an AUC value of 0.981 , indicating that the combination may be a powerful biomarker for TC.

A metagenomic analysis of the stool samples showed that the abundance of Acinetobacter was decreased in patients with colorectal cancer compared with healthy subjects [29]. A 16s rRNA gene sequencing analysis of lung tissues revealed that the microbial community compositions of patients only with emphysema were characterized by a significantly higher abundance of Proteobacteria (primary the genus Acinetobacter) in comparison to lung cancer patients with or without 
emphysema [23]. Acinetobacter is widespread in natural environments and plays an important role in disseminating infections, including the respiratory tract and urinary tract. Certain species of Acinetobacter are resistant to multi-drugs and regarded as important pathogens. Acinetobacter baumannii is an important opportunistic pathogen that is ubiquitous in hospitals and other settings related with healthcare. A systematic review of thirteen original articles showed that gastric carcinogenesis could be associated with an increase in the abundance of Acinetobacter baumannii [30]. These studies showed that the genus Acinetobacter could be related with carcinogenesis of different malignancies. We found that the genus Acinetobacter was enriched in TC tumor tissues, indicating that it may promote TC progression.

Meanwhile, this study has a few limitations. First, the sample size in this study was small. This study has obtained several positive results, which pave the way for future study with larger sample size. Second, this was a retrospective study, and a prospective study is needed to validate our results. Third, the lack of a control group with benign nodules may compromise the interpretation of the results, since comparing the microbial difference of thyroid tissues between patients with benign nodules and TC will further support the observations of this work. In addition, 16s rRNA sequencing can not identify the specific bacterial species, resulting in that information on the species of thyroid microbiota was not obtained. Thus, a study revealing the species of thyroid microbiota is needed in future.

\section{Conclusion}

Taken together, we found that the richness and diversity of thyroid microbiota were significantly lower in TC tumor samples in comparison with matched peritumor tissues. The abundance of genus Sphingomonas and Aeromonas was increased in tumor tissues, while the abundance of Comamonas, Acinetobacter, and Peptostreptococcus was enhanced in peritumor tissues. The combination of Sphingomonas and Comamonas could serve as a powerful marker for distinguishing TC tumor tissues from matched peritumor tissues. Furthermore, the increased abundance of Sphingomonas was correlated with lymph node metastasis, indicating 
that it may play a role in promoting TC development.

\section{Acknowledgements}

The authors would like to thank all patients who participated in this study.

\section{Abbreviations}

ATC: anaplastic thyroid carcinoma; AUC: area under the curve; BMI: body mass index; CI: confidence interval; FTC: follicular thyroid carcinoma; HCTC: Hurthle cell thyroid carcinoma; LEfSe: linear discriminant analysis (LDA) effect size; MTC: medullary thyroid carcinoma; OTU: operational taxonomic unit; PCoA: principal coordinate analysis; PTC: papillary thyroid carcinoma; QC: quality control; ROC: receiver operating characteristic; TC: thyroid carcinoma.

\section{Authors' contributions}

Daofeng Dai: Conceptualization, Methodology, Software, Validation, Investigation, Data curation, Formal analysis, Visualization, Writing-original draft, Writing-review \& editing, Funding acquisition. Yan Yang: Conceptualization, Resources, Investigation. Yong Yang: Conceptualization, Methodology, Software, Validation, Investigation, Data curation, Formal analysis, Visualization, Writing-original draft, Writing-review \& editing. Tianfeng Dang: Investigation, Data curation. Jiansheng Xiao: Supervision. Weibin Wang: Resources, Project administration. Lisong Teng: Conceptualization, Resources, Supervision, Funding acquisition. Jing Ye: Conceptualization, Writing-review \& editing, Supervision, Funding acquisition. Hongqun Jiang: Conceptualization, Writing-review \& editing, Project administration, Supervision. Final approval of manuscript: All authors.

\section{Funding}

This study was supported by the National Natural Science Foundation of China (No. 81860182, 81772853, and 82160527), the Natural Science Foundation of Jiangxi Province (No. 20181BAB205036), Project of the Regional Diagnosis and Treatment Center of the Health Planning Committee (No. JBZX-201903), Science and Technology Department of Jiangxi Province (No. 20203BBGL73201), Health Commission of Jiangxi Province (No. 20203229), and The First Affiliated Hospital of Nanchang University (No. YFYPY202002). 
342 The original data presented in the study are included in the article, further inquiries can be directed

343 to the corresponding authors.

344

345

346

\section{Declarations}

\section{Ethics approval and consent to participate}

This study was conducted in accordance with the code of ethics of the World Medical Association (Declaration of Helsinki) and the studies involving human participants were reviewed and approved by the First Affiliated Hospital, School of Medicine, Zhejiang University, Hangzhou, China. The study was approved by the Medical Research Ethics Committee of the hospital (2020845). The patients provided their written informed consent to participate in this study.

\section{Consent for publication}

All authors give consent for publication.

\section{Competing interests}

The authors have no conflicts of interest to declare.

\section{Author details}

${ }^{1}$ Jiangxi Otorhinolaryngology Head and Neck Surgery Institute, Department of Otorhinolaryngology-Head and Neck Surgery, The First Affiliated Hospital of Nanchang University, Nanchang, China, ${ }^{2}$ Department of Surgical Oncology, The First Affiliated Hospital, School of Medicine, Zhejiang University, Hangzhou, China, ${ }^{3}$ Department of Otolaryngology Head and Neck Surgery, Jiangxi Provincial People's Hospital Affiliated to Nanchang University, Nanchang, China, ${ }^{4}$ Department of General Surgery, the First Affiliated Hospital of Nanchang University, Nanchang, China

\section{References}

[1]Bray F, Ferlay J, Soerjomataram I, Siegel RL, Torre, LA, Jemal, A. Global cancer statistics 2018: GLOBOCAN estimates of incidence and mortality worldwide for 36 cancers in 185 countries. CA Cancer J. Clin. 2018;68: 394-424. 
[2]Pellegriti G, Frasca F, Regalbuto C, Squatrito S, Vigneri R. Worldwide increasing incidence of thyroid cancer: update on epidemiology and risk factors. J. Cancer Epidemiol. 2013;2013: 965212.

[3]Alfano M, Canducci F, Nebuloni M, Clementi M, Montorsi F, Salonia A. The interplay of extracellular matrix and microbiome in urothelial bladder cancer. Nat Rev Urol. $2016 ; 13: 77-90$.

[4]Liu HX, Tao LL, Zhang J, Zhu YG, Zheng Y, Liu D, et al. Difference of lower airway microbiome in bilateral protected specimen brush between lung cancer patients with unilateral lobar masses and control subjects. Int J Cancer. 2018;4:769-778.

[5]Qin J, Li R, Raes J, Arumugam M, Burgdorf KS, Manichanh C, et al. A human gut microbial gene catalogue established by metagenomic sequencing. Nature. 2010;464:59-65.

[6]Iida N, Dzutsev A, Stewart CA, Smith L, Bouladoux N, Weingarten RA, et al. Commensal bacteria control cancer response to therapy by modulating the tumor microenvironment. Science. $2013 ; 342: 967-70$.

[7]Lee YK, Mazmanian SK. Has the microbiota played a critical role in the evolution of the adaptive immune system? Science. 2010;330:1768-1773.

[8]Schwabe RF, Jobin C. The microbiome and cancer. Nat. Rev. Cancer. 2013;13: 800-812.

[9]Fodor AA, Klem ER, Gilpin DF, Elborn JS, Boucher RC, Tunney MM, et al. The adult cystic fibrosis airway microbiota is stable over time and infection type, and highly resilient to antibiotic treatment of exacerbations. PLoS One. 2012; 7: e45001.

[10]Huang YJ, Nelson CE, Brodie EL, Desantis TZ, Baek MS, Liu J, et al. Airway microbiota and bronchial hyperresponsiveness in patients with suboptimally controlled asthma. J. Allergy Clin. 
[11]Sze MA, Dimitriu PA, Hayashi S, Elliott WM, McDonough JE, Gosselink JV, et al. The lung 2012;185:1073-1080.

[12]Hosgood HD, Sapkota AR, Rothman N, Rohan T, Hu W, Xu J, et al. The potential role of lung Mutagen. 2014;55: 643-651.

[13]Ishaq HM, Mohammad IS, Shahzad M, Ma C, Raza MA, Wu X, et al. Molecular Alteration Analysis of Human Gut Microbial Composition in Graves' disease Patients. Int. J. Biol. Sci. 2018; $14: 1558-1570$.

[14]Zhou L, Li X, Ahmed A, Wu D, Liu L, Qiu J, et al. Gut microbe analysis between hyperthyroid and healthy individuals. Curr Microbiol. 2014;69:675-80.

[15]Su X, Zhao Y, Li Y, Ma S, Wang Z. Gut dysbiosis is associated with primary hypothyroidism with interaction on gut-thyroid axis. Clin. Sci. (Lond). 2020;134:1521-1535.

[16]Feng J, Zhao F, Sun J, Lin B, Zhao L, Liu Y, et al. (2019). Alterations in the gut microbiota and metabolite profiles of thyroid carcinoma patients. Int. J. Cancer.2019; 144:2728-2745.

[17]Tudhope, G.R., Wilson, G.M. Anaemia in hypothyroidism. Incidence, pathogenesis, and response to treatment. Q. J. Med. 1960;29:513-537.

[18]Cellini M, Santaguida MG, Virili C, Capriello S, Brusca N, Gargano L, et al. Hashimoto's Thyroiditis and Autoimmune Gastritis. Front. Endocrinol. 2017;8: 92.

[19]Edgar RC, Haas B.J, Clemente JC, Quince C, Knight R. UCHIME improves sensitivity and speed of chimera detection. Bioinformatics. 2011;27:2194-2200. 
412 [20]Edgar RC. UPARSE: highly accurate OTU sequences from microbial amplicon reads. Nat. $413 \quad$ Methods. 2013; 10:996-998.

414 [21]Dhariwal A, Chong J, Habib S, King IL, Agellon LB, Xia J. MicrobiomeAnalyst: a web-based 415 tool for comprehensive statistical, visual and meta-analysis of microbiome data. Nucleic Acids $416 \quad$ Res. 2017;45:W180-188.

417 [22]Liu X, Shao L, Liu X, Ji F, Mei Y, Cheng, Y, et al. Alterations of gastric mucosal microbiota 418 across different stomach microhabitats in a cohort of 276 patients with gastric cancer. EBioMedicine. 2019; 40:336-348.

[23]Liu Y, O'Brien JL, Ajami NJ, Scheurer ME, Amirian ES, Armstrong G, et al. Lung tissue microbial profile in lung cancer is distinct from emphysema. Am. J. Cancer Res.2018; 8: $1775-1787$.

[24]Zhao F, Feng J, Li J, Zhao L, Liu Y, Chen H, et al. Alterations of the Gut Microbiota in Hashimoto's Thyroiditis Patients. Thyroid. 2018; 28, 175-186.

[25]Richard ML, Liguori G, Lamas B, Brandi G, Costa G, Hoffmann TW, et al. Mucosa-associated microbiota dysbiosis in colitis associated cancer. Gut Microbes.2018; 9:131-142.

[26]Jeong JY, Kim TB, Kim J, Choi HW, Kim EJ, Yoo HJ, et al. Diversity in the extracellular vesicle-derived microbiome of tissues according to tumor progression in pancreatic cancer. Cancers (Basel). 2020;12: 2346.

[27]Kanokratana P, Wongwilaiwalin S, Mhuantong W, Tangphatsornruang S, Eurwilaichitr L, Champreda V. Characterization of cellulolytic microbial consortium enriched on Napier grass using metagenomic approaches. J. Biosci. Bioeng. 2018;125:439-447. 
434

435

[28]Ling Z, Shao L, Liu X, Cheng Y, Yan C, Mei Y, et al. Regulatory T Cells and Plasmacytoid Dendritic Cells Within the Tumor Microenvironment in Gastric Cancer Are Correlated With Gastric Microbiota Dysbiosis: A Preliminary Study. Front Immunol. 2019;10:533.

[29]Yang J, McDowell A, Kim EK, Seo H, Lee WH, Moon CM, et al. Development of a colorectal cancer diagnostic model and dietary risk assessment through gut microbiome analysis. Exp. Mol. Med. 2019;51: 1-15.

[30]Dias-Jácome E, Libânio D, Borges-Canha M, Galaghar A, Pimentel-Nunes P. Gastric microbiota and carcinogenesis: the role of non-Helicobacter pylori bacteria - A systematic review. Rev. Esp. Enferm. Dig. 2016;108:530-540. 
Figure legends

FIGURE 1 Flowchart explaining enrollment of patients with thyroid carcinoma for this study.

FIGURE 2 Comparison of microbial profiles between QC samples, TC tumor and matched peritumor tissues before elimination of environmental contamination. (A, B) Chaol and Shannon indices were used to evaluate the microbial diversity of the paired tumor, peritumor tissues, and QC samples. Tumor and matched peritumor tissues were from $30 \mathrm{TC}$ patients. QC samples were used to reveal the environmental microbiota. The Mann-Whitney U test was performed to compare differences between two groups. (C) Principal coordinate analysis (PCoA) of Bray-Curtis analysis demonstrated that QC samples, peritumor and tumor tissues showed three distinct clusters. The microbial relative abundance of TC tumor tissues, matched peritumor tissues, and QC samples at the phylum (D) and genus (E) levels is shown.

FIGURE 3 Comparison of microbial profiles between TC tumor and matched peritumor tissues after elimination of environmental contamination. (A, B) Comparison of Chaol and Shannon indices between $30 \mathrm{TC}$ tumor and matched peritumor tissues after elimination of environmental contamination. (C) Principal coordinate analysis ( $\mathrm{PCoA})$ demonstrated that the peritumor and tumor tissues showed two distinct clusters. The microbial relative abundance of $30 \mathrm{TC}$ tumor tissues and matched peritumor tissues at the phylum (D) and genus (E) levels is shown.

FIGURE 4 The differential taxa at the genus level between the paired thyroid cancer tissues and peritumor tissues from 30 patients with thyroid cancer. (A) Differential 
taxa at the genus level identified by linear discriminant analysis (LDA) effect size (LEfSe) analysis (LDA $>3.0$, corrected $P$ value $<0.05$ ). (B) Differential taxa at the genus level identified by the random forest analysis. (C-G) The differential abundance of the 5 discriminative genera between 30 thyroid cancer tissues and matched peritumor tissues was further validated using the Mann-Whitney U test.

FIGURE 5 Receiver operating characteristic (ROC) curves for differential genera. (A, $\mathbf{B}, \mathbf{C}, \mathbf{D}$, and $\mathbf{E})$ ROC curves for the 5 differential genera and (F) the combination of Comamonas and Sphingomonas were plotted based on microbial relative abundance.

FIGURE 6 The association between clinicopathological factors and thyroid microbiota. (A, B) The differences in Chaol and Shannon indices between tumor tissues from thyroid cancer patients at N0 and N1 stage. Mann-Whitney U tests were performed. (C) Principal coordinate analysis (PCoA) based on Bray-Curtis distance revealed that thyroid cancer patients at N0 stage were significantly different from those at N1 stage. (D) Linear discriminant analysis (LDA) effect size (LEfSe) analysis (LDA $>3.0$, corrected $P$ value $<0.05$ ) was performed to evaluate differential taxa at the genus level. (E) The differential abundance of Sphingomonas between N0 and N1 stage was further validated using the Mann-Whitney $U$ test. (F) The receiver operating characteristic (ROC) curve for Sphingomonas genera was plotted using microbial relative abundance to assess the value of thyroid microbiota as a diagnostic tool to distinguish between thyroid patients with N0 and N1 stage. $(\mathbf{G}, \mathbf{H})$ The differences in Chaol and Shannon indices between tumor tissues from male and female patients with thyroid cancer. Mann-Whitney U tests were performed. (I) 
500 Principal coordinate analysis (PCoA) of thyroid microbiota in male and female 501 patients with thyroid cancer based on Bray-Curtis distance. $(\mathbf{J}, \mathbf{K})$ The differences in 502 Chao1 and Shannon indices between tumor tissues from thyroid cancer patients aged $503 \geq 55$ and $<55$. Mann-Whitney $U$ tests were performed. (L) Principal coordinate 504 analysis (PCoA) of thyroid microbiota in patients with thyroid cancer aged $\geq 55$ and $<$ 50555 based on Bray-Curtis distance.

508 Table 1. Clinical features of patients with thyroid carcinoma. 
Figures

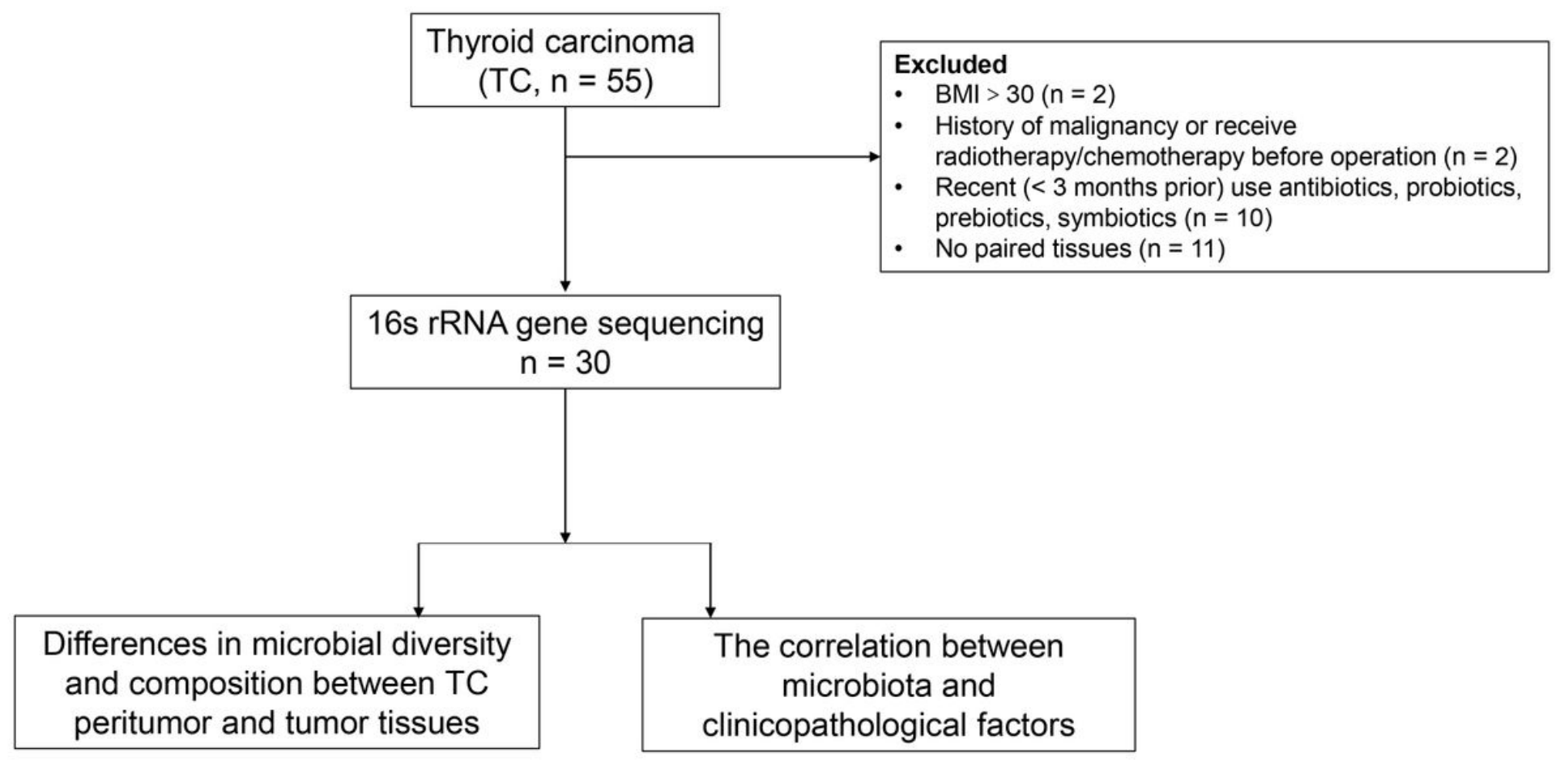

\section{Figure 1}

Flowchart explaining enrollment of patients with thyroid carcinoma for this study 

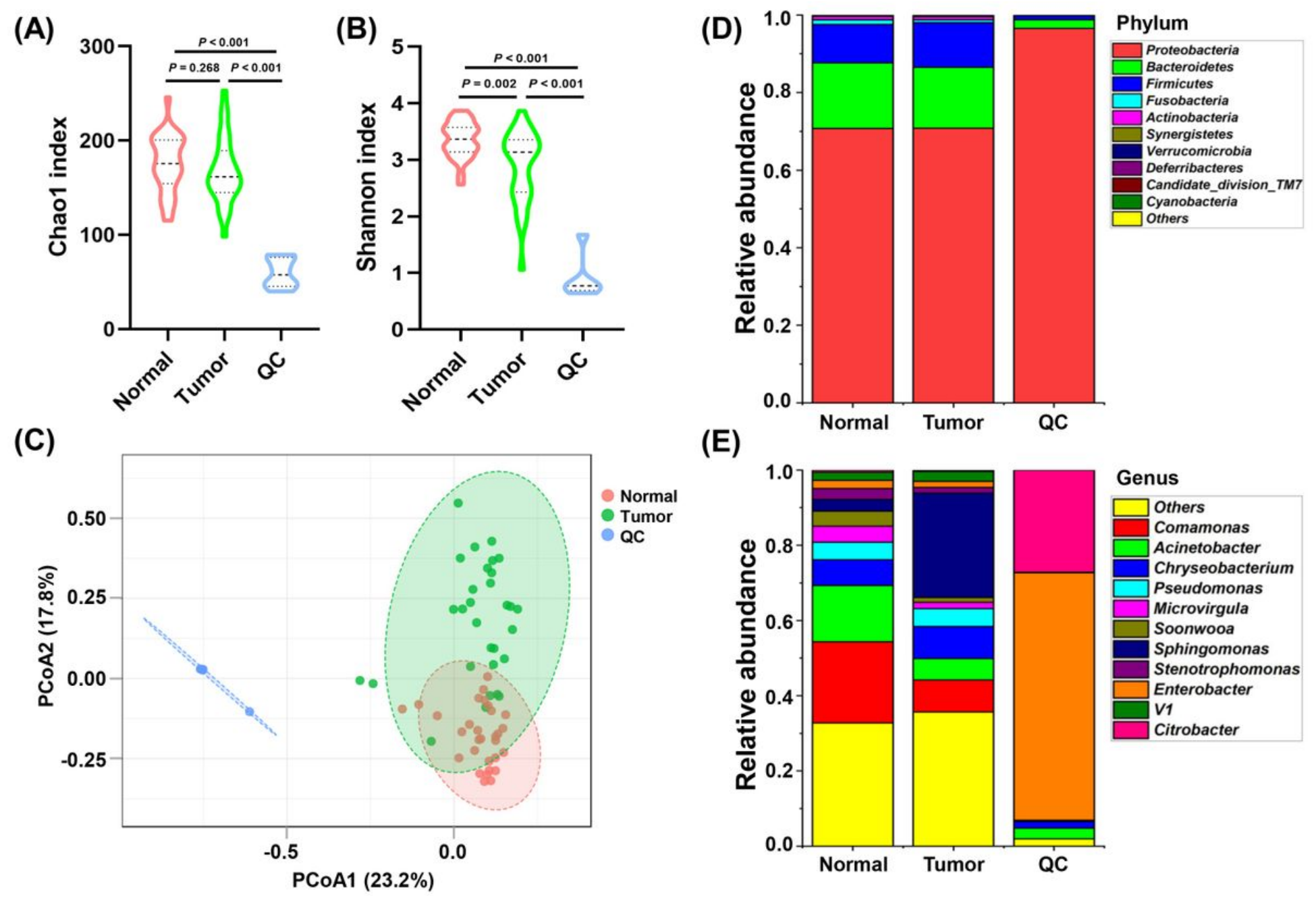

(E)

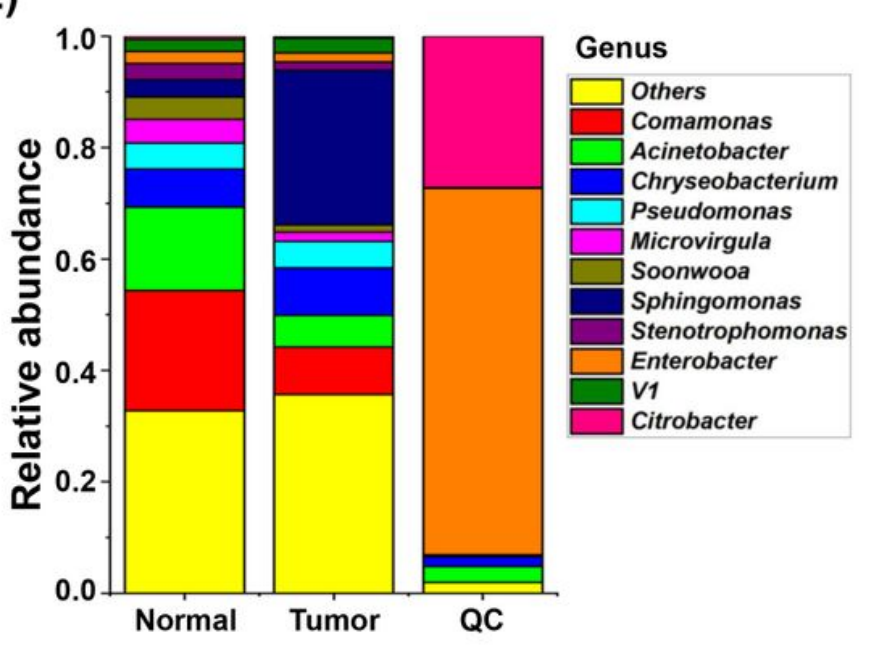

Figure 2

Comparison of microbial profiles between QC samples, TC tumor and matched peritumor tissues before elimination of environmental contamination. (A, B) Chao1 and Shannon indices were used to evaluate the microbial diversity of the paired tumor, peritumor tissues, and QC samples. Tumor and matched peritumor tissues were from 30 TC patients. QC samples were used to reveal the environmental microbiota. The Mann-Whitney $U$ test was performed to compare differences between two groups. (C) Principal coordinate analysis (PCoA) of Bray-Curtis analysis demonstrated that QC samples, peritumor and tumor tissues showed three distinct clusters. The microbial relative abundance of TC tumor tissues, matched peritumor tissues, and QC samples at the phylum (D) and genus (E) levels is shown. 
(A)

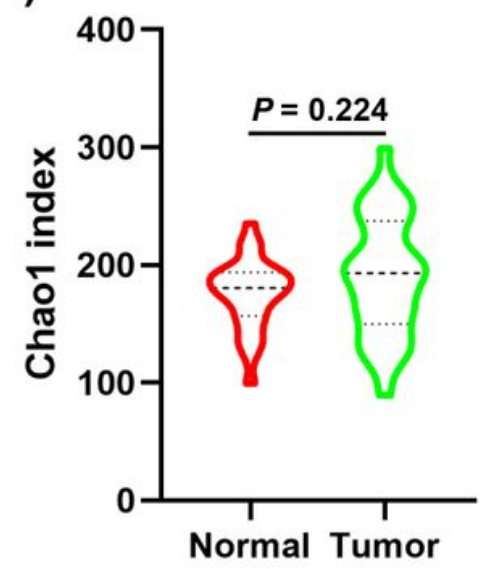

(B)

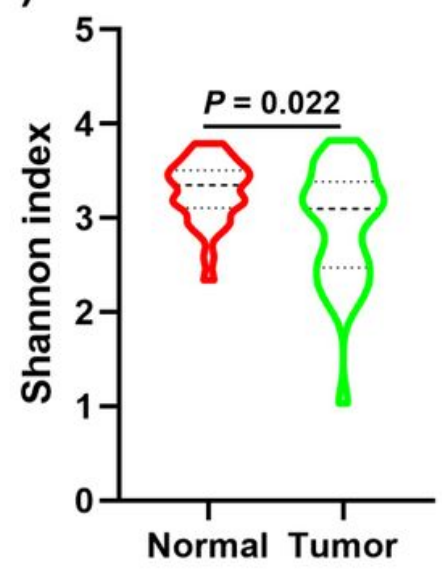

(D)

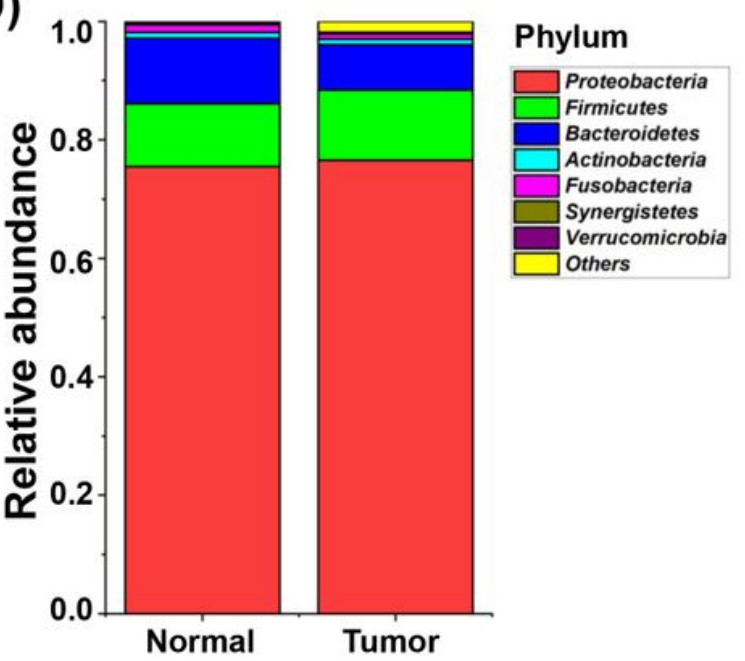

(E)
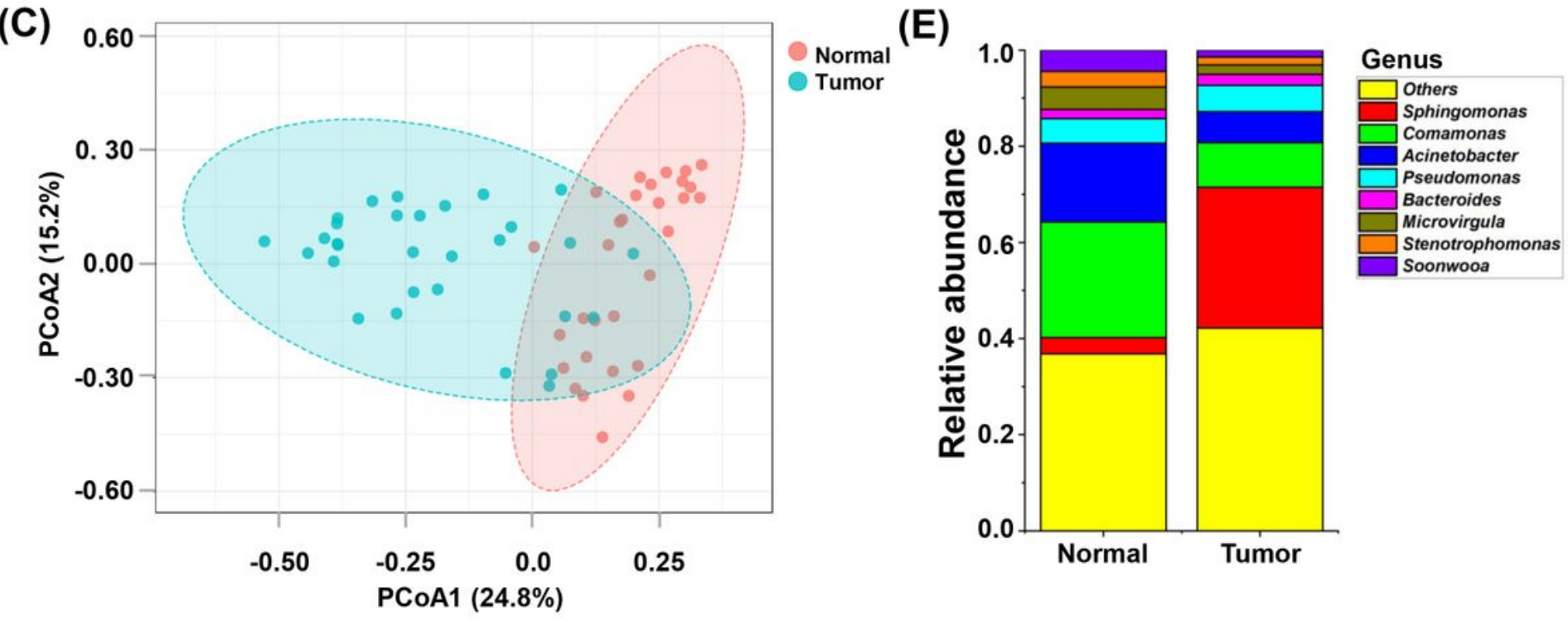

Figure 3

Comparison of microbial profiles between TC tumor and matched peritumor tissues after elimination of environmental contamination. (A, B) Comparison of Chao1 and Shannon indices between 30 TC tumor and matched peritumor tissues after elimination of environmental contamination. (C) Principal coordinate analysis (PCOA) demonstrated that the peritumor and tumor tissues showed two distinct clusters. The microbial relative abundance of $30 \mathrm{TC}$ tumor tissues and matched peritumor tissues at the phylum (D) and genus (E) levels is shown. 
(A)

(B)

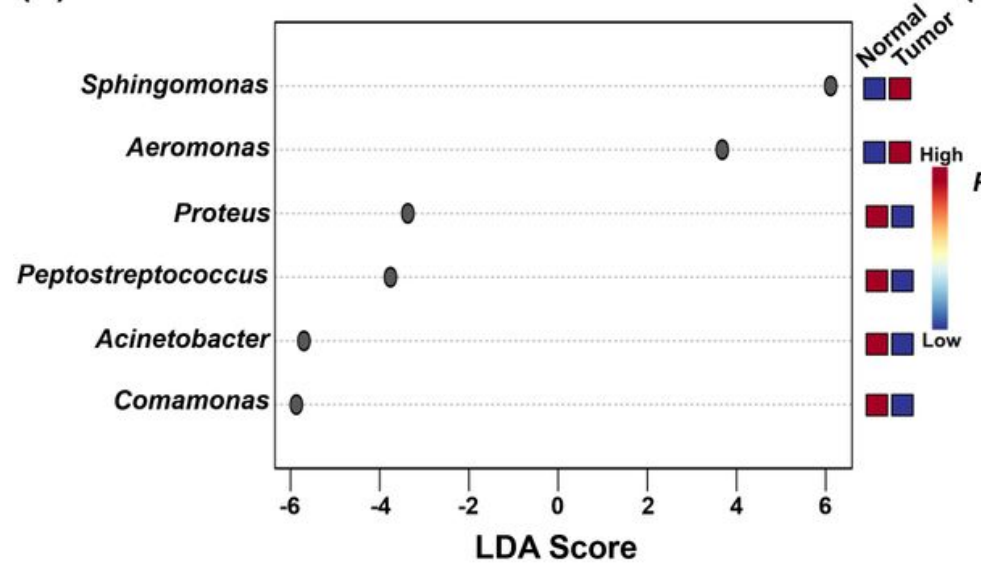

(C)

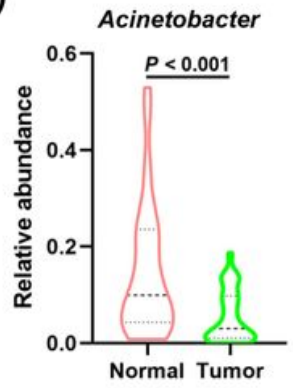

(D)

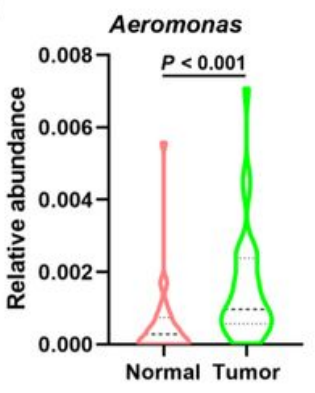

(E)

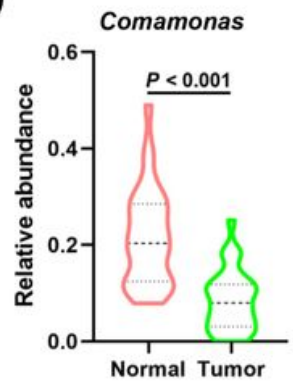

Sphingomona

Comamonas

Acinetobacter

Peptostreptococcus

Shewanel

Aeromonas 。

Morganella •

Delftia •

Lautropia

Dysgonomonas

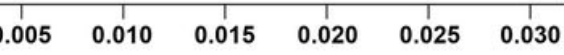

Mean Decrease Accuracy

(F)

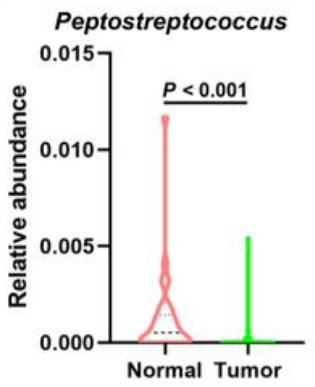

(G)

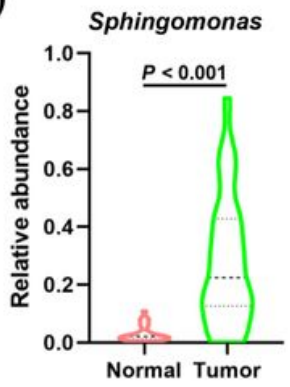

Figure 4

The differential taxa at the genus level between the paired thyroid cancer tissues and peritumor tissues from 30 patients with thyroid cancer. (A) Differential taxa at the genus level identified by linear discriminant analysis (LDA) effect size (LEfSe) analysis (LDA > 3.0, corrected $P$ value $<0.05$ ). (B) Differential taxa at the genus level identified by the random forest analysis. (C-G) The differential abundance of the 5 discriminative genera between 30 thyroid cancer tissues and matched peritumor tissues was further validated using the Mann-Whitney U test. 
(A)

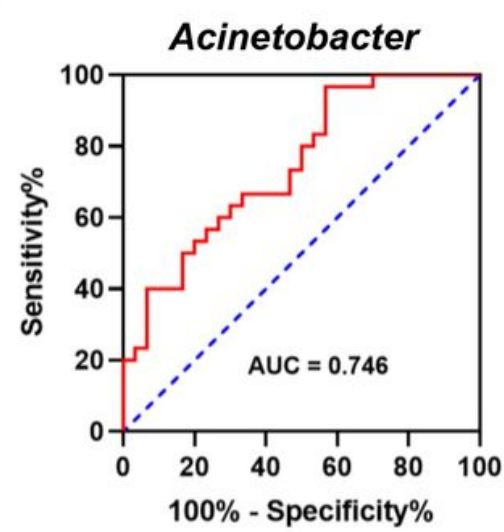

(D)

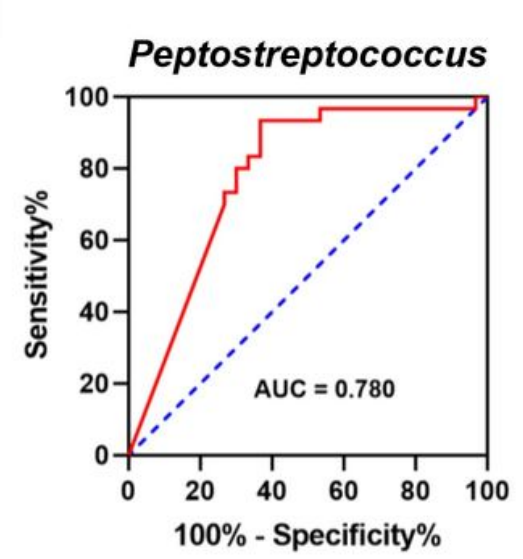

(B)

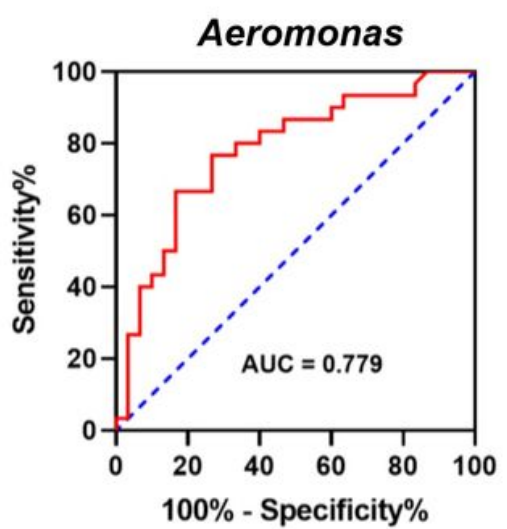

(E)

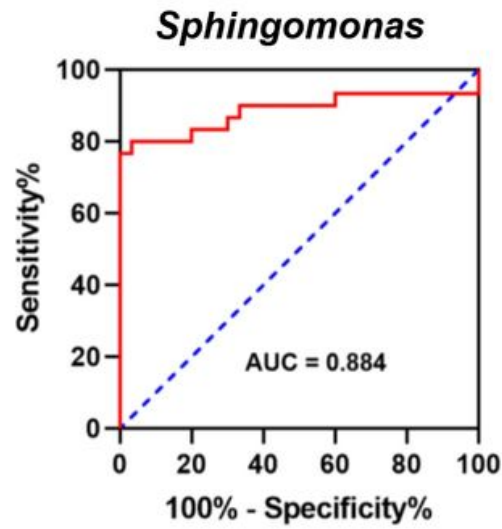

(C)

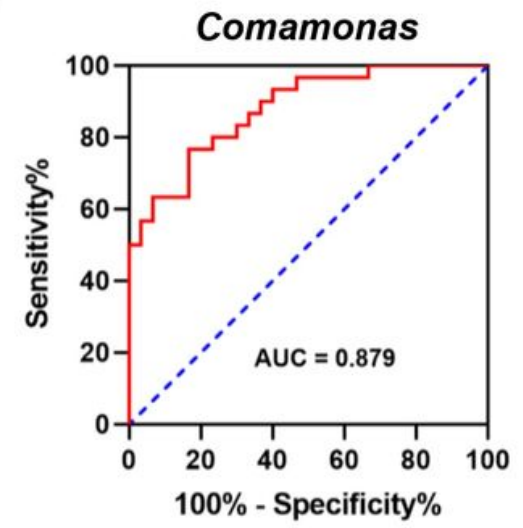

(F)

Comamonas and Sphingomonas

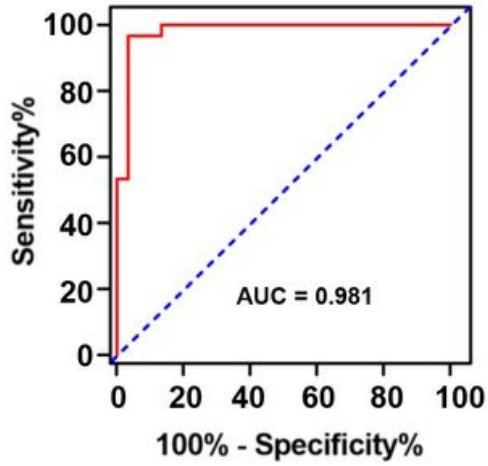

Figure 5

Receiver operating characteristic (ROC) curves for differential genera. (A, B, C, D, and E) ROC curves for the 5 differential genera and $(F)$ the combination of Comamonas and Sphingomonas were plotted based on microbial relative abundance. 
(A)

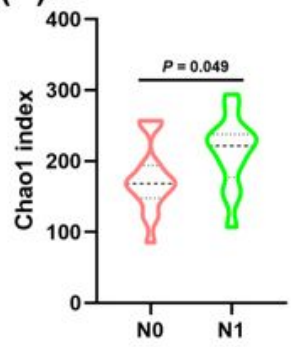

(C)

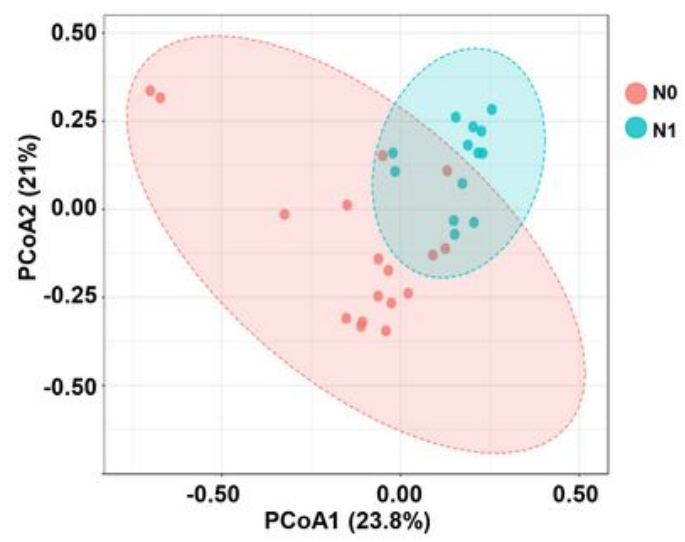

(G)

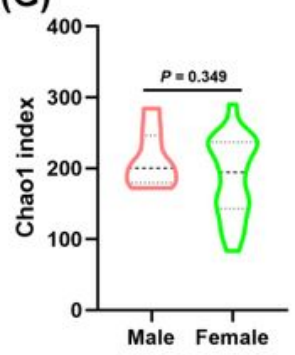

(I)
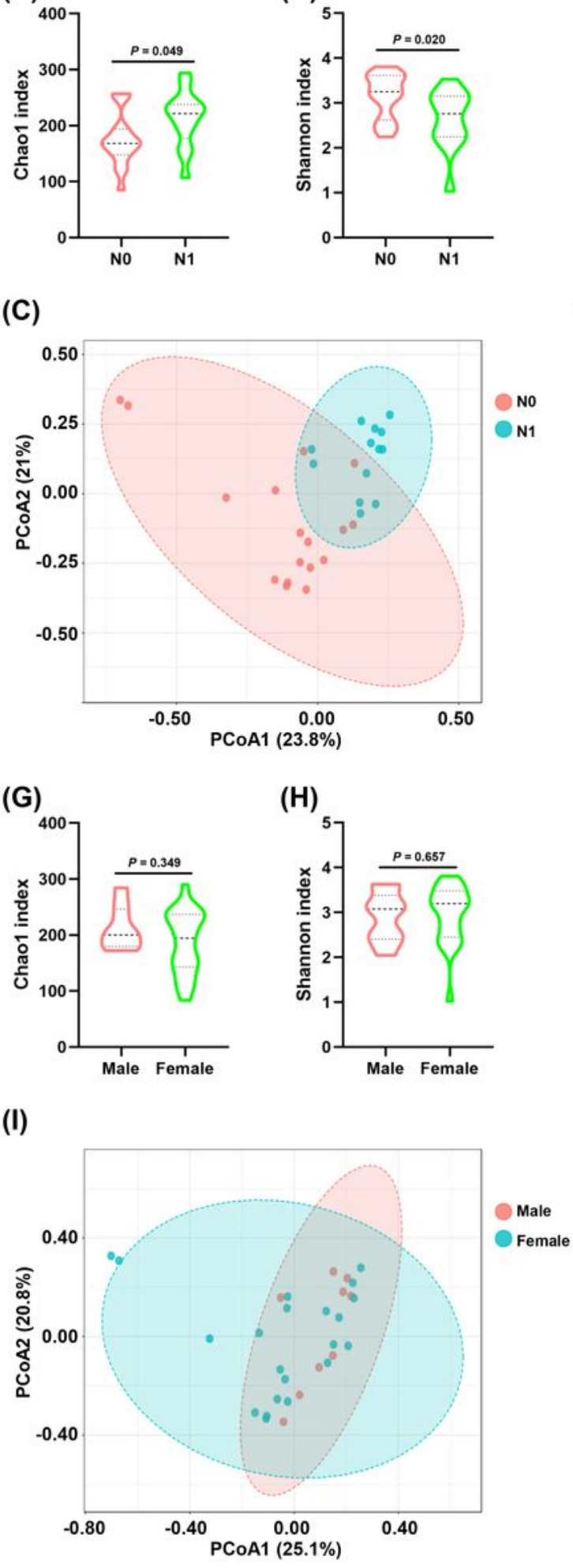

(D)

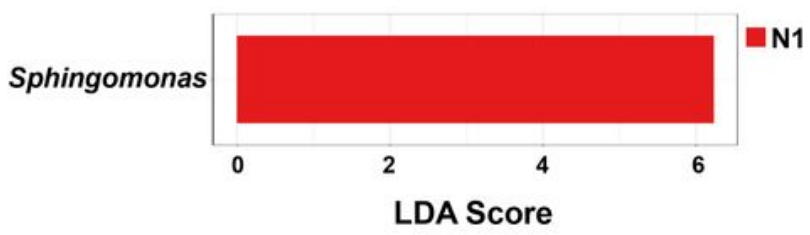

(E)

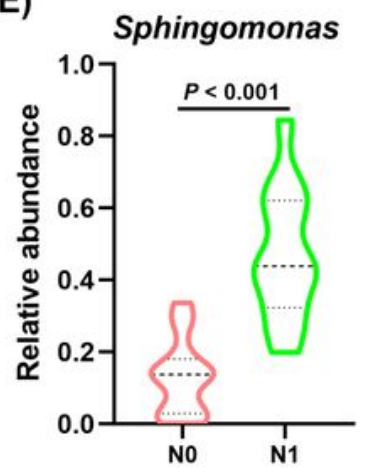

(F)

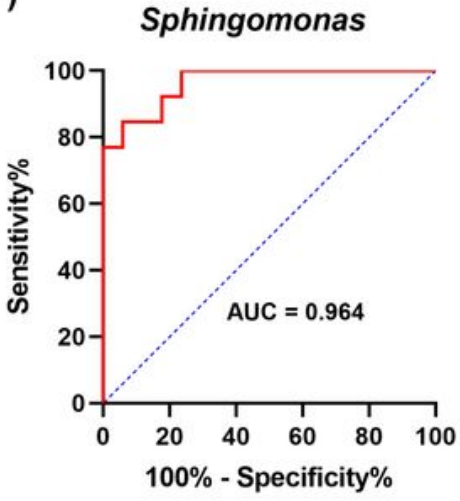

(J)

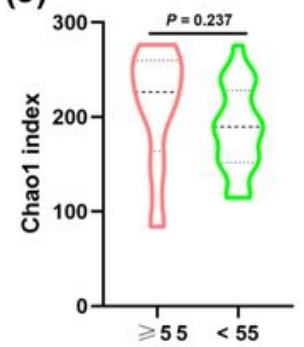

(K)

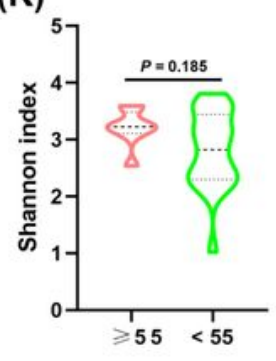

(L)

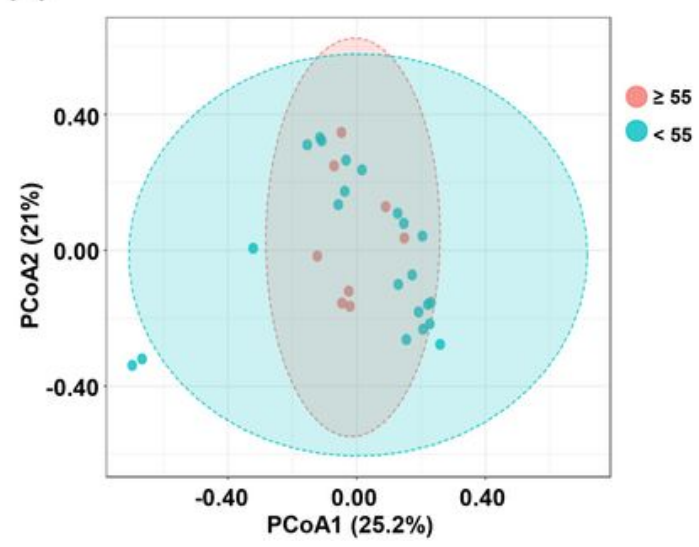

Figure 6

The association between clinicopathological factors and thyroid microbiota. (A, B) The differences in Chao 1 and Shannon indices between tumor tissues from thyroid cancer patients at N0 and N1 stage. Mann-Whitney $U$ tests were performed. (C) Principal coordinate analysis (PCoA) based on Bray-Curtis distance revealed that thyroid cancer patients at N0 stage were significantly different from those at N1 stage. (D) Linear discriminant analysis (LDA) effect size (LEfSe) analysis (LDA > 3.0, corrected P value < 
0.05) was performed to evaluate differential taxa at the genus level. (E) The differential abundance of Sphingomonas between N0 and N1 stage was further validated using the Mann-Whitney U test. (F) The receiver operating characteristic (ROC) curve for Sphingomonas genera was plotted using microbial relative abundance to assess the value of thyroid microbiota as a diagnostic tool to distinguish between thyroid patients with N0 and N1 stage. $(G, H)$ The differences in Chao1 and Shannon indices between tumor tissues from male and female patients with thyroid cancer. Mann-Whitney $\mathrm{U}$ tests were performed. (I) Principal coordinate analysis (PCOA) of thyroid microbiota in male and female patients with thyroid cancer based on Bray-Curtis distance. (J,K) The differences in Chao1 and Shannon indices between tumor tissues from thyroid cancer patients aged $\geq 55$ and $<55$. Mann-Whitney $U$ tests were performed. (L) Principal coordinate analysis (PCoA) of thyroid microbiota in patients with thyroid cancer aged $\geq 55$ and $<55$ based on Bray-Curtis distance.

\section{Supplementary Files}

This is a list of supplementary files associated with this preprint. Click to download.

- TableS1.docx

- Tables2.csv

- TableS3.csv

- Tables4.csv

- Tables5.csv 